\title{
A Proposal To Enhance Cellular And Wifi INTERWORKING TOWARD A RELIABLE HETNET
}

\author{
Nam Nguyen and Takuro Sato \\ Graduate School of Global Information and Telecommunication Studies \\ Waseda University, Tokyo, Japan
}

\begin{abstract}
WiFi offloading is becoming one of the key enablers to help the network operators dealing with the exponentially growing demand of mobile data. The idea of using WiFi to offload data traffic from cellular network has proposed for many years. However, the interoperability issue between the two networks needs to be enhanced so that WiFi can efficiently supplement for the cellular network in case of congestion or outage. In this paper, we propose a novel network roaming and selection scheme based on 3GPP TS 24.312 and IEEE $802.11 \mathrm{k}$, u standards to enhance cellular and WiFi interworking. The proposed scheme is aimed at enhancing the network roaming and selection so that WiFi network can serve as a supplement and backup access network for the cellular not only for congestion control but also in case of unexpected network failure event. We also model and evaluate the proposed scheme in a typical HetNet with interworking WiFi access points and cellular base stations. The simulation result shows that our proposed scheme quickly detects unexpected network failure event and assists active UEs to perform handoff to preferable alternative point of access. As a result, service disruption is substantially reduced and quality of experience (downlink/uplink's throughput) is improved. Therefore, our proposed scheme can be used for a more reliable HetNet in terms of congestion control and disruption tolerance.
\end{abstract}

\section{KEYWORDS}

WiFi cellular interworking; reliable HetNet; congestion control; network failure tolerance.

\section{INTRODUCTION}

In recent years, the proliferation of smart devices (smart phones and tablets) and mobile Internet applications is causing the explosion of mobile traffic. Experts forecast that mobile data traffic will increase more than ten times by 2020 [1], whilst billions of devices will be interconnected with mobile broadband connections by then. This massive increase of mobile data traffic raises a major concern for network operators who own limited radio spectrum. Since the carrier's network infrastructure cannot measure up with the growth of mobile traffic, network congestion is happening more frequently, especially in crowded area. In addition, in many areas, the wireless network is vulnerable to natural disasters or outage, which can cause unexpected service disruption to the mobile network. For these reasons, increasing network capacity and resilience become major concerns for network operators.

Offloading mobile traffic to other networks is one of the key solutions for aforementioned problem. Offloading is referred as using alternative network technologies for delivering data that is originally targeted for, e.g., using WiFi to offload traffic from cellular network when it becomes congested. It helps to ensure optimal usage of available radio resources and load balancing among available radio accesses. 
The obvious choices for offloading are small cells and Wi-Fi. However, as far as we concerned, more and more network operators commit to adopt $\mathrm{WiFi}$ as the extended access for cellular network. Unlike any other small cell technologies (femto-cell and pico-cell), WiFi utilizes unlicensed frequency band causing minimum interference to macro-cell. Besides, WiFi can cut down the CAPEX for operators since it is cheaper and easier to deploy in large scale especially in indoor environment such as airport, shopping mall or any other crowded area. In case of network outage, WiFi can serve as a backup network for cellular network since it is supported by wide varieties of portable devices such as smartphones and tablets. For these reasons, WiFi is emerging to become one of the key enablers to deal with congestion issue of future cellular network.

Over the past years, 3GPP has been working on new functionality that will allow Wi-Fi AP to connect on the Evolved Packet Core (EPC), which is the core network of 3GPP 4G network and future wireless network. As a result, the operators are able to offer a carrier grade Wi-Fi that allows the cellular users to offload part of their traffic. The interworking issue between 3GPP cellular and non-3GPP access network has been discussing by both 3GPP and IEEE. For instance, 3GPP proposed a new entity so called Access Network Discovery and Selection Function (ANDSF) or TS 24.312 [3] as well as mobility management such as GTP, Proxy IP. On the other hand, IEEE ratified a new enhancement for IEEE 802.11 specification to enhance WiFi and cellular interworking known as IEEE 802.11u [4] or Hotspot 2.0 (HS 2.0) [5]. These are the key enablers to make smooth and seamless roaming from non-3GPP to 3GPP cellular network.

Although the key enabler standards are available, the effective way of combining them to make a reliable wireless network is out of scope of these standards. This is the next big challenge in WiFi and cellular interoperability. Indeed, with existing standards, it is possible to make a carrier grade Wi-Fi so that cellular users can enjoy all of cellular services while they roam to WiFi. However, the biggest challenge is how to assist the mobile node to intelligently discover and select the most preferable Point of Service at anytime and anywhere so that users can use the best access network for different individual data service. This issue is also important to network operators because they want to steer the UE's traffic among available access networks intelligently to optimize wireless resource and prevent congestion. Furthermore, proactively steering traffic between available access technologies can increase the resilience and reliability of the network in case of emergency. For example, when the 4G macro-cell base station is not accessible due to disaster or blackout, the system must quickly find and transfer active mobile-nodes to available point of service to minimize service disruption. Because WiFi is versatile and easily to deploy, it is less vulnerable to unexpected network failure.

The main drivers for the WiFi-cellular network interworking are to reduce the load on the cellular network and backup for each other in case of unexpected network failure event. Therefore, while several radio accesses are available, the assigning and properly making handoff decision among access networks to avoid congestion become the fundamental issue. Furthermore, when one access network is no longer available due to network failure or outage, it is desirable to assign active users to alternative available point of service as fast as possible. While there are only a limited number of studies so far considering this issue, the evaluation of proposed method has also been neglected or insufficiently provided. In this paper, based on state of the art 3GPP and IEEE standards, we propose a novel monitoring and roaming selection scheme to enhance interoperability of cellular and $\mathrm{WiFi}$, specifically for congestion-controlled and resilient HetNet.

By taking advantage of ANDSF's discovery and selection policies and real-time network condition (Signal Strength and Access Point's load), the proposed scheme decides right timing for mobile nodes to make macro-cell and WiFi roaming decision for traffic congestion avoidance and unexpected network failure event. For evaluation, we develop our system model of ANDSF [3], IEEE 802.11k [4] standards and perform simulation of the proposed scheme in a typical HetNet 
scenario with macro-cell and WiFi interworking. Unlike previous works, our simulation model is an end-to-end model from application to physical layer. We take into account user 's mobility and realistic traffic model. During the simulation process, the ANDSF server and the UE's connection manager entities behave and interact corresponding to real-time network events. The simulation result shows that our proposed scheme effectively assists the UE to make macro-cell and WiFi roaming decision when cellular network failure occurs namely, disrupted connection event. The proposed scheme is compared with conventional WiFi steering strategy (WiFi always has the highest priority and WiFi selection is based on RSS/SINR), which is adopted by most of existing smart devices. By using the proposed scheme, the UE quickly detects unexpected network event and selects the preferable point of service so that service disruption time is reduced and UE's Quality of experience (downlink/uplink throughput) is less affected. In addition, the proposed scheme also prevents network congestion by dynamically steering active UEs to available point of services.

\section{BACKGROUND AND RELATED WORKS}

\subsubsection{GPP ACCESS NETWORK Discovery AND SELECTION FunCTION}

3GPP has considered the non-3GPP and 3GPP cellular network interworking issues since 3GPP release 8 [2]. It proposed a tight-coupling All-IP architecture that integrates non-3GPP radio access with 3GPP core network so called EPC (Evolved Packet Core) [2]. In addition, a new entity, Access Network Discovery and Selection Function (ANDSF) is standardized in the 3GPP TS 23.312[3], which contains data management and control functionality to provide necessary access network discovery and selection assistance to the UE as per the operator's policy. From the beginning, the ANDSF is standardized as an entity, through which the network operator can provide inter-system mobility policies. It is a 3GPP approach for controlling offloading between 3GPP and non-3GPP access networks. The purpose of the ANDSF is to assist user devices to discover access networks in their vicinity and to provide rules (policies) to prioritize and manage connections to all networks.

Researches on the ANDSF's functions and its role have not finalized, further enhancements and extensions of ANDSF are expected in future $3 \mathrm{GPP}$ releases.

In essence, ANDSF serves as the network discovery and selection policy repository server located at the EPC of the network provider. It communicates with the UEs over the standardized S14 interface. This interface (defined in TS 23.402 [2]) enables dynamic provision of information to the UE for access network discovery and selection classified as three set of information:

- Intersystem Mobility Policy (ISMP)

- Access Network Discovery Information

- Inter System Routing Policy (ISRP)

This information is to provision the UE a set of rules to select preferable point of service as well as routing policies for multi-homing UE. Upon provisioned with these rules, the UE continuously checks the validity of the rules and selects the valid one with the highest priority. Validity can include date, time of day, location.

\subsection{IEEE STANDARDS FOR 3GPP INTERWORKING}

IEEE is actively working on non-3GPP and 3GPP interworking issues, especially WiFi and 3GPP interworking. It ratified an amendment for IEEE 802.11 standard known as IEEE802.11u [4]. The aim of this amendment is to make roaming from WiFi to 3GPP cellular network as smooth and seamless as roaming inside cellular network. 
The next generation of WiFi access point also known as Hotspot 2.0 (HS2.0) [5] (mainly based on IEEE802.11u) will be available in near future. Beyond the security authentication enhancements, the main features of HS2.0 Release 1[5] are new enhancements to facilitate AP discovery and selection functions. With the Access Network Query Protocol (ANQP) and the Generic Advertisement Service (GAS), various types of network information can be provided to UE before association such as real-time load of the AP, authentication type, supported Downlink/Uplink throughput etc.

\subsection{RELATED WORKS}

The conventional WiFi offloading strategy (adopted by most of smart device) is that WiFi always has higher priority than $3 \mathrm{G} / 4 \mathrm{G}$ cellular. As a result, the UE switches to WiFi whenever AP is detected. Besides, WiFi AP selection is simply based on the Receive Signal Strength (RSS) or SINR, thus the nearest AP is selected. However, we believe that RSS or SINR information alone is not enough to make intelligent WiFi selection, especially in place, where there are collocated APs at the same area. In such scenario, the selection scheme that is solely based on RSS can lead to bad quality of experience (QoE) for UE or network resource underutilization issue. Therefore, we need to take into account other factors to enhance roaming and point of service selection decision. Handoff decision based on multiple inputs such as bandwidth, QoS, cost of service have been proposed for years with complex models and algorithms such as fuzzy MADM, TOPSIS, SAW, ELECTRE, AHP [6]. However, the authors failed to describe how their proposal could be implemented in practice. Since their proposals are required expensive computation cost and multiple metrics (service cost, available bandwidth, device's speed, jitter), which are difficult to obtain from existing standards. In [7], 4GAmericas provided a comprehensive view of the state of the art of the key enablers for integrating cellular and WiFi. It explained how combining the advantages of ANDSF and HS2.0 could resolve the problems of macro-cell and WiFi roaming.

However, there was no detail description, numerical evaluation such as simulations or experiment's result provided. In [8] the authors examined the mobility between different access technologies in wireless networks and focus on the case of interoperability. The quality of the service of mobility, the time required for the handover and the existing loss of packages were also extensively analysed. In [9] authors summarized recent developments in the field of delay and disruption tolerant networking. Numerous implementations and applications have emerged with a wide variety of performance and application domains were highlighted in the works. In [10] authors highlighted technical challenges in heterogeneous wireless networks underlying seamless vertical handover. The authors also presented a detailed survey on the vertical mobility management process with a focus on decision-making mechanisms. The importance of multihoming in a heterogeneous environment was also pointed out in this work. There are also some researches on LTE-Advanced, which try to increase the cooperation among neighbour LTE based stations for load-balancing and enhancing resilience in homogeneous LTE network [11]. This approach is also known Self Organizing Network (SON). In [12] the authors addressed solutions for WiFi offloading in LTE cellular networks when demands exceed the capability of the LTE access. They compared the performance of each access technology using different network performance metrics. In [13], the authors proposed a novel network-assisted user-centric WiFioffloading model for maximizing per-user throughput in a heterogeneous network. In the proposed model, the network collects network information, such as the number of users in WiFi networks and their traffic load. Then, the network decides the specific portion of traffic to be transmitted via WiFi networks so that the individual user's throughput can be maximized by offloading more traffic to WiFi. Through analysis, the authors investigated the effect of the WiFioffloading ratio on the per-user throughput. However, they did not consider user's mobility in their simulation. In [14], the authors studied how much economic benefits can be anticipated thank to delayed WiFi offloading method, by modelling the interaction between a single provider 
and users based on a two-stage sequential game. They first analytically proved that WiFi offloading is economically beneficial for both the provider and users. Their major focus was to understand how and how much users and the provider obtained the economic incentives by adopting delayed WiFi offloading and investigated the effect of different pricing and delaytolerance. In [15], the authors investigated the performance improvement induced by adopting a hybrid cellular/ WiFi communication architecture where the mobile users can be served by either the LTE eNodeB or a mobile WiFi AP. In the proposed scheme, the mobile WiFi APs were considered as relay entities wirelessly connected to the LTE eNodeB and shared this broadband connection with other users over WiFi tethering (using WiFi frequencies). Important performance metrics of the proposed hybrid scheme including the average bit error probability (ABEP), capacity and outage probability were theoretically studied.

The IEEE 802.21 in [16], Media Independent Handover (MIH) is a standardized framework proposed by IEEE, which facilitates vertical handover across heterogeneous networks. It defines a generic media- independent-handoff (MIH) framework to support information exchange between network elements as well as a set of functional components to execute the roaming process. IEEE 802.21 specifies three media-independent services. Media-Independent Event Service (MIES), Media Independent Command Service (MICS), Media-Independent Information Service (MIIS).

The MIIS provides a data store of available networks and network parameters, and defines standard query/response messages to access and retrieve such information for each available access network. The MIIS's role is similar to ANDSF. In [17, 18] provided an overview of vertical handoff approaches based on MIH framework. The work presented comprehensive solutions to ensure VHO between three types of different RATs: Wi-Fi, WiMAX and 3G, dealing with multiple parameters to make VHO decision. To the best of our knowledge, in recent years, researches related to MIH is gradually fading out from the academic studies and there is no initiative from industries to implement this standard in commercial products. The reason is that it is required many changes and modifications to the MAC layer of existing IEEE 802.11, 802.16, 802.3 standards as well as 3GPP LTE, overall core network architecture and protocols. In addition, the main function, MIIS service for network discovery can be replaceable by ANDSF, which is more robust and popular. For this reason, we consider using ANDSF instead of MIH to provide discovery and selection policy utilized in our scheme.

\section{Proposed Dynamic Wifi-3GPp RoAming Decision Scheme}

\subsection{Proposal Motivations}

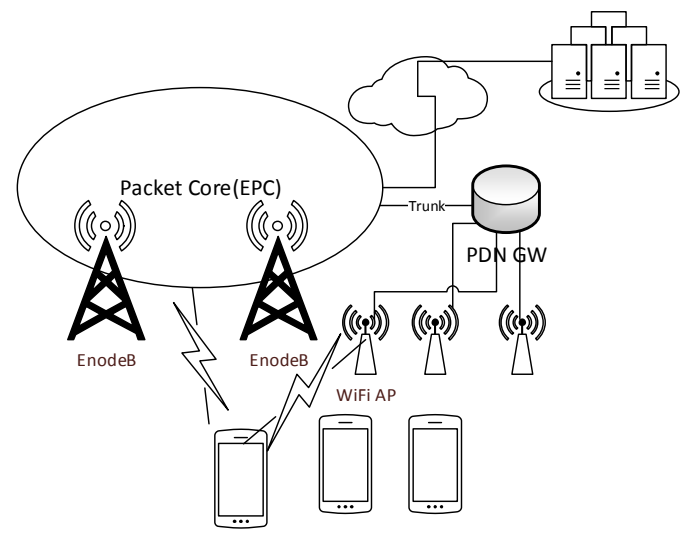

Figure 1. Heterogeneous network scenario with LTE cellular and WiFi interworking. 
Our considering scenario is that a network operator provides high-speed mobile data service via both cellular (LTE, WiMAX) and WiFi networks. The cellular and WiFi access networks are connected with a common core network. The scenario is also extended to separated cellular carriers and $\mathrm{WiFi}$ providers as long as they have interworking agreement and physically interconnected network. As we anticipate that high speed data service via multiple access networks will become norm in near future due to exponential growing demand of mobile data while licensed frequency resource is very limited.

Figure.1 shows the baseline scenario when a user moves from LTE network to a WiFi network coverage. It is worth noting that a smooth and seamless transition between LTE and WiFi is not in the scope of this paper. The mobile nodes can freely decide their preferable access network and APs. Depending on preferences, the UEs may switch access from LTE to WiFi and vice versa.

Whenever WiFi access network is available nearby, the operator can choose to offload some or all of its traffic through WiFi AP depending on the network condition. This selectively offloading traffic to legitimated WiFi networks gives mobile operators an opportunity to increase their total network capacity to meet rising traffic demands and a way to extend network coverage and capacity to WiFi networks. For such scenario, the ANDSF [3] framework is a well suitable candidate since it is a $3 \mathrm{GPP}$ approach for controlling handover operation between 3GPP and non3GPP access networks. Since release 8 of 3GPP, 3GPP has specified the ANDSF framework through which the network operator can provide a list of preferred access networks with intersystem mobility policies. However, it is proved that relying sole on ANDSF is not enough [12], UE needs to take into account the real time network performance to make handoff decision when the condition is favourable. For example, offloading to WiFi network should only carry out when the cellular congestion is getting worse and the available AP guarantees acceptable QoS. In addition, WiFi can also serve as a backup access for cellular in case of network failure and vice versa. For instance, the traffic of impaired cellular nodes can be steered to available point of service in order to keep the network service running with low or possibly even no negative impact to end user's experience.

Therefore, we need more robust network roaming decision and selection scheme to enhance cellular and WiFi interworking. Because we aim to make the new scheme should be pragmatically implementable, we take advantage of ratified 3GPP and IEEE standards with minimum modification.

\subsection{ANDSF WLANSP Management ObJect ANd Proposed New Enhancement}

As we aforementioned, in a heterogeneous wireless network with cellular and WiFi interworking, it is desirable to have an entity from network side to regulate the UEs when and where to make cross network roaming decision. Besides, this entity should also provide rightful selection rule for UEs. For instance, only legitimate AP (AP belongs to operator or operator who has roaming agreement) should be selected. These are main functions of ANDSF described in 3GPP documents [3]. However, as described in [3], the ANDSF's selection policy for access network is rather static, which is not helpful for dynamic congestion control or network failure occurrence. For example, the operator can predefine the priority for each WiFi AP or BS according to integer number from 1-255 (one is the highest priority). Therefore, the one with higher priority should be selected first. However, the condition of the AP/BS may change dynamically from time to time.

Therefore, if the UE only relies on the ANDSF selection rule, it might be degraded the end-user experience in certain cases (The AP is too far away, or it is overloaded). In our proposal, ANDSF's selection policies is used to help the UE obtain available and legitimate AP's list. It can also control the number of UE that can roaming to WiFi by providing load and RSS thresholds. 
In the latest 3GPP release 12, the TS 24.312 [3] which is a tree based Management Object of discovery and selection policy repository, a new leaf of ANDSF Management Object (ANDSF MO) is defined for WiFi Selection called WLANSP (WLAN Selection Policy). The WLANSP information can contain a set of one or more WLANSP rules. Each rule can have one or more WiFi selection criterion defined in ANDSF/WLANSP/ $/ \mathrm{X}>/$ SelectionCriteria node including:

- CriteriaPriority.

- HomeNetworkIndication.

- PreferredRoamingPartnerList.

- MinBackhaulThreshold.

- MaximumBSSLoadValue.

- RequiredProtoPortTuple.

- PreferredSSIDList.

- SPExclusionList.

The UE can optionally obtain these selection criteria and use them for Wi-Fi selection procedure.

If the UE does not provision with these criteria, the conventional selection strategy will be applied. However, in our proposed scheme, for the sake of simplicity, we only consider the MaximumBSSLoadValue criterion for Wi-Fi AP selection because it can serve as a load threshold for our selection scheme. If the channel load of the candidate AP does not satisfy this threshold, it will be eliminated from the selection process.

Apart from the load metric threshold, we consider the receive signal strength (RSS) as a factor that can affect the UE's experienced QoS. Therefore, in our proposed scheme, we propose to append a signal level threshold to eliminate far away candidate AP. However, the signal level threshold (minimum signal strength) is not included in the ANDSF OM TS 23.312[3]. We proposed to include this parameter in the ANDSF OM WLANSP leaf so that UE can easily fetch this information from ANDSF server. Therefore, we propose to append this value to the same OM leaf of MaximumBSSLoadValue in ANDSF WLANSP MO, $<\mathrm{X}>/$ WLANSP $/<\mathrm{X}>/$ SelectionCriteria/ $<\mathrm{X}>/$ MinimumBSSRSSValue so that this value can be delivered to UE in the same manner. The RSS and load thresholds are using in our scheme to control the number of UEs can roaming. For example, if this load threshold value is low, only certain number of UEs can roam to WiFi so that the load threshold condition can be satisfied. If the RSS threshold is high, only near enough UEs can access. Figure 2 shows how this parameter can be added to existing ANDSF MO specification.

\subsection{Real-Time Channel Load Metric}

According to IEEE 802.11u [4] and HS.2.0 standard [5], the channel load is defined as one of the new QoS metric appended to beacon message of next generation of WiFi AP. The channel load indicates the real-time load condition of the WiFi AP. The channel load metric is first proposed in IEEE 802.11k, e [19]. This is IEEE 802.11's amendment for WiFi Radio Resource Management and quality control. The channel load indicates the available channel resource of a WiFi AP. We select the load metric as the criterion for AP selection because it is evident that if the load of AP is too high, it can greatly degrade active mobile node's QoS.

According to [19], the channel utilization or channel load, $U_{c}$ is defined as the percentage of time, linearly scaled with 255 representing $100 \%$, that the AP sensed the medium was busy, as indicated by either the physical or virtual carrier sense (CS) mechanism. Therefore, this metric indicates the real-time load status of AP radio channel. The channel load is calculated by 
(Equation 1), The Channel_Busy_Fraction, $t_{\text {busy }}$ is defined as the number of microseconds while the physical or virtual carrier sense mechanism in MAC layer keeps indicating busy channel status. The Measurement_Duration $T_{M}$ represents the number of consecutive beacon intervals while the channel busy time is measured. $t_{c}$ is calculated by (Eq.2), is the channel utilization measured interval representing the number of consecutive beacon intervals during which the channel busy time is measured. $t_{b}$ is the beacon period value in WiFi IEEE802.11time unit (TU). One TU is 1024 microseconds.

$$
\begin{aligned}
& U_{c}=\text { Integer }\left(\frac{t_{\text {busy }}}{T_{M}}\right) \times 255 \\
& T_{M}=t_{c} \cdot t_{b} \cdot 1024
\end{aligned}
$$

The channel load $U_{c}$ is an important QoS metric to select preferable WiFi AP in our proposed scheme.

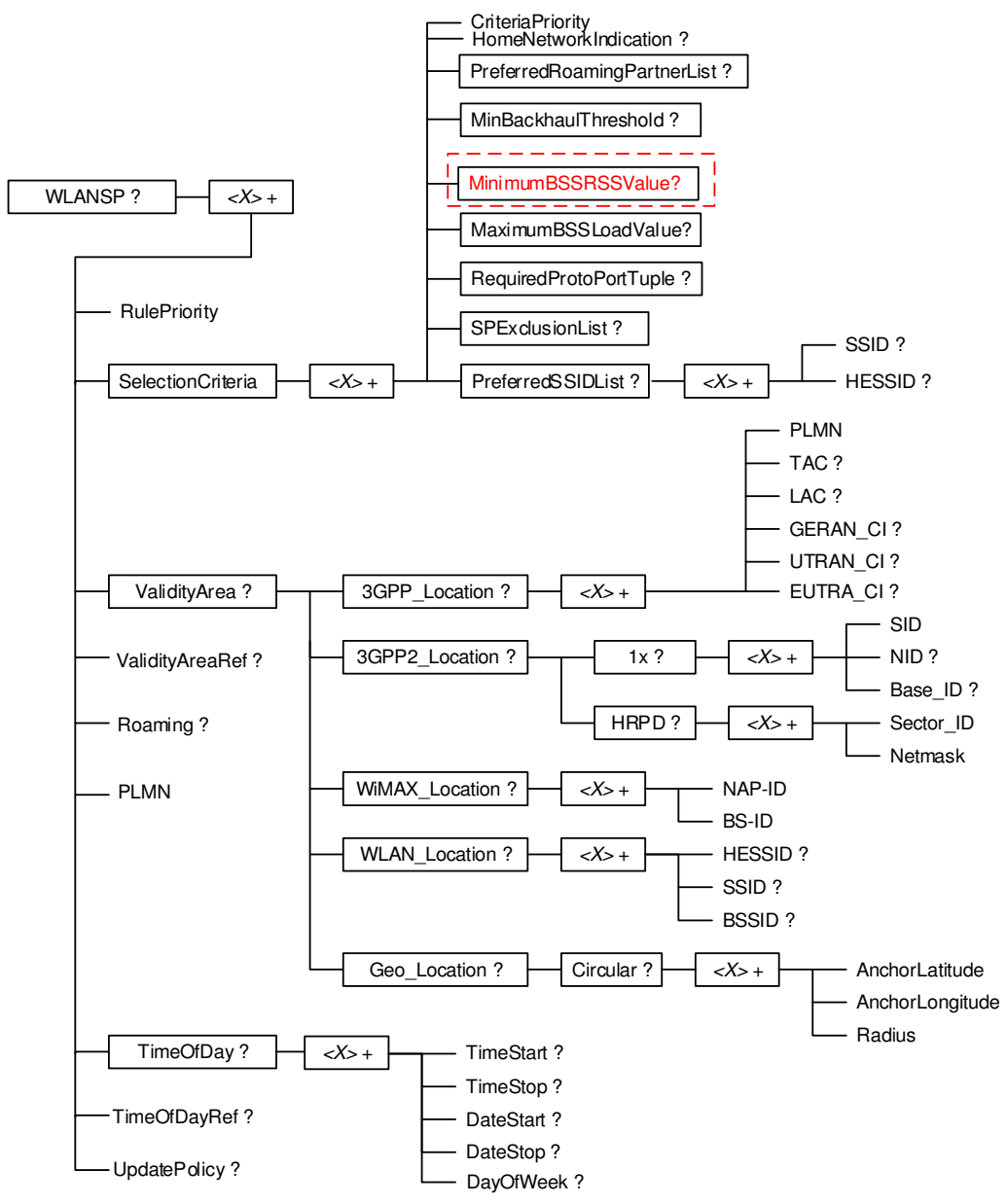

Figure 2. Example of enhanced ANDSF OM for WLANSP. 


\subsection{RECEIVE SignAL STRENGTH}

In the proposed scheme, we also consider the Receive Signal Strength, RSS as one of input for WiFi selection. The RSS is the most widely used input for conventional handoff decision since it is easy to measure and directly relates to the radio channel quality. With stronger receive signal strength, the AP can use high performance channel modulation and coding scheme and the throughput will become higher accordingly.

$$
\overline{R S S}_{i}=\alpha \cdot R S S_{i}+(1-\alpha) \cdot \overline{R S S}_{i-1}
$$

$\overline{R S S}_{i}$ is the average of RSS which is calculated as (Equation 3), where $\alpha$ represents the weighted value. In this equation, although the range of value is $0-1$, typically the variable is 0.75 or above. $\overline{R S S}_{i}$ represents the most recently measured value, and $\overline{R S S}_{i-1}$ represents the previous averaged value. This approach ensures that a large difference in RSSI values will be smoothed. The purpose is to reduce the fluctuation of measured value obtained from the physical layer due to channel fading or interference.

\subsection{Proposed Monitoring And Wlan-3Gpp Roaming Decision Scheme}

In this subsection, we present our proposed monitoring and roaming decision scheme to enhance the interworking between $\mathrm{WiFi}$ and cellular network. The proposed scheme is a decision-making procedure carried out by the connection manager (CM) of end user's device. Since the UE has a unique position that allows it to receive both the operator's defined policies and real-time network condition of surrounding area. Therefore, it is preferable to let the UE make roaming and selection decision instead of any other entity in the network. Therefore, the proposed scheme is a network assisted and host-controlled model. It is worth noting that we do not consider handoff between macro-cell BSs since it is well specified in 3GPP Standard documents. The proposed scheme should not interfere with macro-cell handoff strategy. Figure. 3 describes the flow chart of proposed scheme:

Step 1: The CM monitors the QoS and status current connection. If the QoS of connection is degrading, (namely the load of associated AP exceeds certain threshold), better QoS is requested by running applications, current condition is suddenly disrupted, it will trigger the process to find alternative point of service.

Step 2: The CM attempts to contact the ANDSF server to retrieve the list of legitimate WiFi APs corresponding to UE's location (either Cell-ID, or SSID or GPS location if applicable) or time. In case of connection failure, the UE can utilize the last provisioned data from ANDSF. In our proposal, the ANDSF policies is used to shorten the scanning process for UE in the next step. If the ANDSF's policies is not available, the UE will have to scan all WiFi channels to find available APs.

Step 3: By using AP discovery information from ANDSF, the UE only scans the indicated WiFi channels to check whether the candidate APs are available because provisioned ANDSF's information may be out of date or unreachable from UE's location. By doing this step, UE eliminates unreachable AP and obtains the RSS and the channel load of each candidate AP. We assume that the candidate APs support either HS 2.0 or IEEE $802.11 \mathrm{k}$, e. so that the real-time load and the RSS of each AP can be obtained from the beacon message or via ANQP protocol in case of HS.2.0 [5]. 
Step 4: The CM obtains the list of APs that fulfil the load and RSS thresholds. These thresholds can be specified by network carrier via ANDSF's WiFi selection policies [3]. As aforementioned, the MaximumBSSLoadValue can be used to select WiFi AP, if the load of AP is higher than this value, it will be eliminated. We introduce the load threshold in order to pre-eliminate unsuitable AP, which cannot guarantee a good QoS. The MinimumBSSRSSValue can serve as the RSS threshold to eliminate far away AP candidates. Therefore, only candidates that satisfy channel load and RSS criteria are selected. However, these thresholds can be adaptively decreased in the extreme case when current connection is disrupted and none of candidate can be found. For example, MaximumBSSLoadValue can be gradually decreased up to $90 \%$ while MinimumBSSRSSValue can be reduced to 0.9*Received Signal Sensitive that is the lowest received signal strength that can sustain a normal transmission. If the thresholds are decreased to their minimum value and no candidate can be found, the $\mathrm{CM}$ will increase delay counter to start over from step 1.

Step 5: If the number of the qualified AP in step 4 is only one, the qualified AP will be selected as the candidate for WiFi roaming. However, if the number of qualified AP is more than one, the UE's CM will sort the candidates based on their load, RSS and ANDSF's priority properties. Ranking based on load, RSS metrics has higher priority than ANDS's policy. The AP that has the highest ranking is selected. The CM starts the handoff procedure to target AP.

Step 6: if there is no better candidate AP in terms of channel load and RSS criteria, the UE will stay at the current point of service. The UE's CM returns to step one and start over if the QoS is still not acceptable or network disruption event is triggered; if there is no candidate found in last attempt, the retry time will be increased. Therefore, we can reduce unnecessary attempt when there is no better point of service can be found.

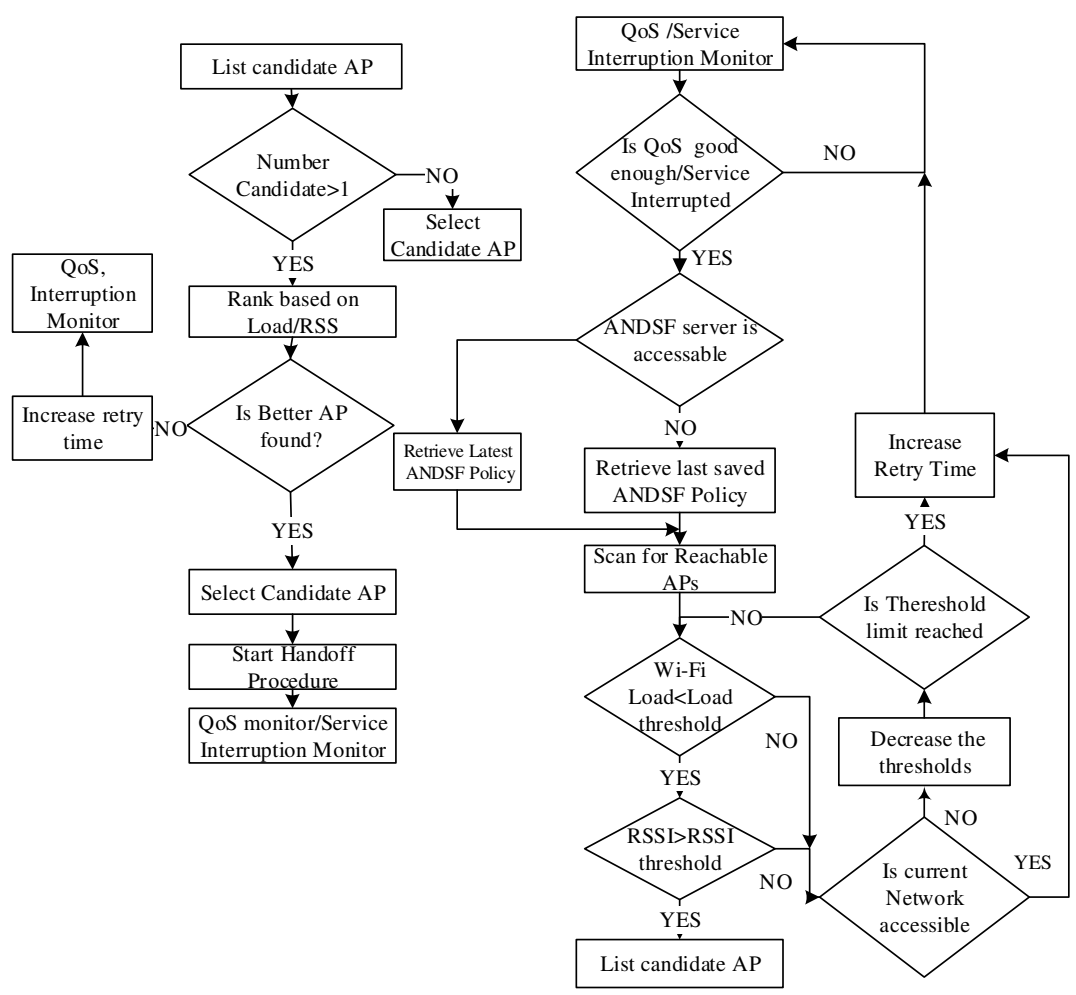

Figure 3. Proposed Monitoring and WLAN-3GPP roaming decision scheme. 
International Journal of Computer Networks \& Communications (IJCNC) Vol.8, No.1, January 2016

The pseudo code for our proposed scheme is shown as follows:

Algorithm Roaming decision and WiFi AP selection Scheme

Input: Set required QoS, current AP

Output: AP candidate.

1. While $(1)$ do \{

2.if QoS degrading or Current connection drop trigger then

3.Obtain data from ANDSF server

a. Obtain AP candidates Information (SSID, operating channel)

b. Obtain Load and RSS thresholds MaximumBSSLoadValue, MinimumBSSRSSValue

4. qualified_AP_List $=$ nil;

for each $A P_{i}$ in $A P$ candidate List 1

a. Obtain information of available $A P_{i}$ information from physical Layer,

b. Obtain $R S S_{i}$, calculate $\overline{R S S}_{i}$ Equation(1).

c. Obtain channel_load $_{i}$ information.

d. if $\overline{R S S}_{i}>$ MinimumBSSRSSValue \&\& channel_load $_{i}<$ MaximumBSSLoadValue then

qualified_AP_List add $A P_{i}$

\}

If qualified_AP_List is empty\&\& Current connection drop then \{

MinimumBSSRSSValue= MinimumBSSRSSValue.0.9;

MaximumBSSLoadValue=0.9;

Goto Step 4;

\}

If qualified_AP_List is empty $\& \&$ MaximumBSSLoadValue $==0.9$ then return;

5. Candidate_AP=AP

for each $A P_{i}$ in $A P$ candidate qualified_AP_List \{

if $\left(A P_{i}->C h a n n e l \_l o a d<C\right.$ Candidate_AP->Channel_load $)$ then Candidate_AP $=A P_{i}$;

else if $\left(A P_{i}->\right.$ Channel_load== Candidate_AP- $>$ Channel_load $)$ then \{

$\operatorname{if}\left(A P_{i} \rightarrow \overline{R S S}>\right.$ Candidate_AP-> $\left.\overline{R S S}\right)$ then Candidate_AP $=A P_{i}$;

\}

\}

6. if Candidate_AP $!=$ current $A P$ then return Candidate_AP;

\} 


\section{Simulation AND RESUlt}

\subsection{Simulation SETUP}

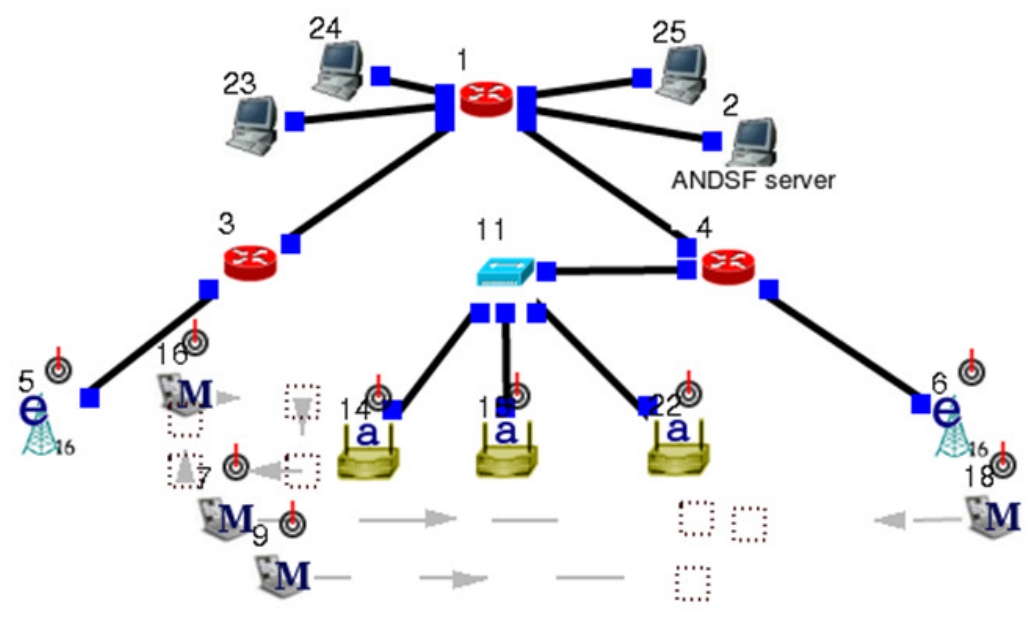

Figure 4. Simulation setup with 2WiMAX BS, 4Mobile Nodes, ANDSF server, Correspondence Nodes.

Although there are some practical implementations of ANDSF TS 24.312[3] from the industries [20], as far as we concerned, there is no publicly disclosed simulation model of ANDSF for academic study. Therefore, in order to evaluate our proposal, we model the ANDSF TS 24.312[3] in a typical heterogeneous network scenario with interworking macro-cell BS and WiFi APs. The system model is built on NCTUns 6.0, which is powerful open source network simulator developed by National Chiao Tung University [21, 22, 23, 24]. It can simulate almost all of popular wired and wireless nodes and protocols such as IEEE $802.11 \mathrm{a} / \mathrm{b} / \mathrm{g}$, ad-hoc networks, WiMAX IEEE $802.16 \mathrm{~d} / \mathrm{p}$ BS, multiple wireless interface UE, GPRS, satellite and vehicular networks .etc. Moreover, one of the unique features of this simulator is that it allows running $\mathrm{C} / \mathrm{C}++$ application on the simulated node. In our simulation model, we take advantage of this feature to simulate the ANDSF server and the CM for each mobile node. ANDSF server is a $\mathrm{C}$ program, which provisions information of available APs, load threshold, RSS threshold.

This information can be retrieved by any UE by pull method. The CM is also $\mathrm{C}$ program running on each mobile node executes our proposed scheme. It can obtain the RSS and the real-time channel load of AP candidate through MAC layer. We use COST 231 model for radio propagation model so that the RSS of AP can be derived from UE via Physical Layer and MAC layer. As the simulation tool does not support IEEE802.11k, u, we modify the MAC layer of the WiFi AP node to append the real-time channel load information into the beacon message. In Equation (1), the channel busy fraction is defined as the number of microseconds in which the physical or virtual carrier sense indicates the channel is busy. In our simulation, we use the virtual carrier sense, Request to Send/Clear to Send (RTS/CTS) mechanism to detecting busy channel status. In [19], the default value of the channelUtilizationBeaconIntervals in (1) is set to 50.

Although the NCTUns simulator supports multiple interface mobile node and horizontal handover (handover within one access network), it does not support handoff between WiFi and macro-cell or vertical handoff (VHO). Therefore, we modify the source code to simulate the vertical handover between macro-cell (WiMAX BS) and WiFi AP. It is worth noting that our 
proposed scheme is proposed for 3GPP Cellular (LTE or UMTS) and Wi-Fi. However, the simulation software NCTUns version 6.0 [18] does not support LTE. Therefore, we have to use WiMAX instead of LTE for our HetNet model. Since our proposed scheme does not consider any input from the macro-cell, using WiMAX access instead of LTE does not cause any difference in the simulation result. Due to a problem related to Mobile IP module of the simulator [21], the simulation of VHO is not a seamless handover. As a result, UE's connection is interrupted during the VHO.

In our simulation model, we evaluate how our proposed scheme performs when unexpected network failure event occurs e.g. macro-cell base station connection is suddenly disrupted. We compare the result with the conventional case when conventional WiFi selection is applied. The criteria to evaluate are the service disruption time and the throughput of UE in such event.

\subsection{SiMUlATION DESCRIPTION}

Figure.4 shows the screenshot of our simulation setup in the simulator and the simulation parameters are listed in Table I. This setup is a typical WiFi deployment scenario to offload traffic from cellular network as we described in Figure 1. We arrange a typical WiMAX-WiFi interworking scenario with two IEEE 802.16e BS1, 2 (Node 5, 6), three 802.11a WiFi AP 1, 2, 3 (Node 14, 15, 22) and four multiple wireless interface UE 1, 2, 3, 4 (Node 7, 9, 16, 18 consecutively) equipped with both WiMAX and WiFi 802.11a interfaces. It is worthy to note that the node IDs are assigned automatically by the simulation software. We rename them according to this convention for coherence purpose. The UEs move under the coverage of BS1, 2 toward WiFi coverage area. All of the UEs have a CM to monitor and control their connectivity. The ANDSF server (Node2) is located at network carrier's side and reachable by any UEs regardless of their point of service. Its role is to provide WiFi candidate APs as well as load and RSS threshold information when UEs move to one particular location.

At the beginning, the UEs are associated with macro cell; UE 1, 2, 3 associated with BS1; UE4 associated with BS2. At $\mathrm{T}=5 \mathrm{~s}$, the UEs start sending greedy CBR (Constant Bit Rate) traffic toward the Correspondent Nodes $(\mathrm{CN}) \mathrm{s}$ (node 23, 24, 25). Figure. 4, 5, 6, 7 show the throughput of UE 1, 2, 3, 4 gradually increase from $\mathrm{T}=5 \mathrm{~s}$ and reach climax at $640 \mathrm{~KB} / \mathrm{s}$.

At $\mathrm{T}=16$ (s), the BS1 and associated UEs connection are disrupted. This is to simulate the unexpected network failure event when macro-cell BS is temporary disrupted and only WiFi APs are available. The Figure 5, 6, 7 (red line) show the throughput of UE 1, 2 and 3 dropping abruptly from $\mathrm{T}=16 \mathrm{~s}$. The CM of UEs detect the connection failure and execute the proposed roaming decision procedure. The CM of UEs retrieve information from ANDSF server containing a list of candidate AP1, 2, and 3 (Node 14, 15, 22). The UEs scan the surrounding area based on the indicated channel. Since there are 3 candidate APs available, they are ranked based on channel load, RSS, ANDSF's priority. The UE1, UE2, UE3 choose AP1, AP2, AP3 for handoff and start handoff procedure to targeted APs respectively. Figure. 9 shows the UEs complete the handoff procedure and start sending traffic through corresponded APs. Figure [5-7] show the throughput of $\mathrm{UE} 1,2,3$ raising from $\mathrm{T}=20$ s because in our simulation model, the VHO procedure takes approximately 4 seconds to complete.

At T=25s, the connection between BS2 (Node 6) and UE4 (Node 18) is also purposely disrupted to simulate network failure. Figure. 8 shows the traffic of UE4 dropped rapidly from T=25s. The CM of UE4 carries out similar procedure to handoff to candidate AP3. The CM of UE4 selects and starts VHO procedure to AP3. Figure.8 shows that the traffic of UE4 going through AP3 starts rising from T=30s. In Figure. 6, 7, 8 (the red line), we notice that the throughput of UE2 is dropped to $435.66 \mathrm{~KB} / \mathrm{s}$ while the throughput of UE4 only reaches $573.804 \mathrm{~KB} / \mathrm{s}$ at $\mathrm{T}=30 \mathrm{~s}$. The reason is that UE2, 3, 4 are connected to AP3 sharing $9 \mathrm{Mb} / \mathrm{s}$ bandwidth of AP3. 
International Journal of Computer Networks \& Communications (IJCNC) Vol.8, No.1, January 2016

Table 1. Simulation Parameters

\begin{tabular}{|c|c|c|c|}
\hline \multicolumn{3}{|c|}{ WIMAX } & Wi-Fi \\
\hline Technology & \multicolumn{2}{|c|}{ IEEE $802.16 \mathrm{e}$} & IEEE 802.11a \\
\hline Coverage & \multicolumn{2}{|l|}{$1000 \mathrm{~m}$} & $50 \mathrm{~m}$ \\
\hline RX Thresh & \multicolumn{2}{|l|}{$-96 \mathrm{dBm}$} & $-82 \mathrm{dBm}$ \\
\hline Transmit Power & \multicolumn{2}{|l|}{$35 \mathrm{dBm}$} & $16.02 \mathrm{dBm}$ \\
\hline Channel & \multicolumn{2}{|l|}{5,6} & $1,2,4$ \\
\hline Bandwidth/QoS & \multicolumn{2}{|l|}{$5 \mathrm{Mb} / \mathrm{s}$} & $9 \mathrm{Mb} / \mathrm{s}$ \\
\hline Modulation scheme & \multicolumn{2}{|c|}{ OFDM 16QAM } & OFDM 16QAM \\
\hline Carrier frequency & \multicolumn{2}{|l|}{$2.3 \mathrm{GHz}$} & $5 \mathrm{G} \mathrm{GHz}$ \\
\hline ChannelUtilizationBeaconIntervals & \multicolumn{2}{|l|}{ NA } & 10 \\
\hline BS Number & \multicolumn{2}{|l|}{2} & 3 \\
\hline Propagation channel & \multicolumn{2}{|c|}{ Two-ray ground } & Two-ray ground \\
\hline \multicolumn{4}{|c|}{ MS } \\
\hline \multicolumn{2}{|l|}{ Node number } & \multicolumn{2}{|c|}{4} \\
\hline \multicolumn{2}{|l|}{ Mobility movement } & \multicolumn{2}{|c|}{ Straight line } \\
\hline \multicolumn{2}{|l|}{ Multiple interface } & \multicolumn{2}{|c|}{ WIMAX and Wi-Fi } \\
\hline \multicolumn{2}{|l|}{ Speed } & \multicolumn{2}{|c|}{$2-5 \mathrm{~m} / \mathrm{s}$} \\
\hline \multicolumn{2}{|l|}{ Traffic parameter } & \multicolumn{2}{|c|}{ Greedy CBR } \\
\hline \multicolumn{4}{|l|}{ Miscellaneous } \\
\hline \multicolumn{2}{|l|}{ Channel load broadcast interval } & \multicolumn{2}{|l|}{$1 \mathrm{~s}$} \\
\hline \multicolumn{2}{|l|}{ Channel load threshold } & \multicolumn{2}{|l|}{0.8} \\
\hline \multicolumn{2}{|l|}{ RSS load threshold } & \multicolumn{2}{|c|}{$0.8^{*} \mathrm{RX}$ Thresh } \\
\hline
\end{tabular}

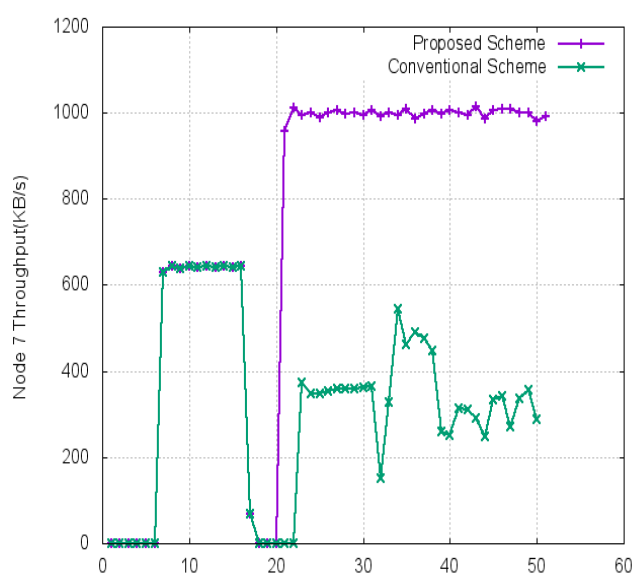

Figure 5. Throughput of UE1 (Node 7)

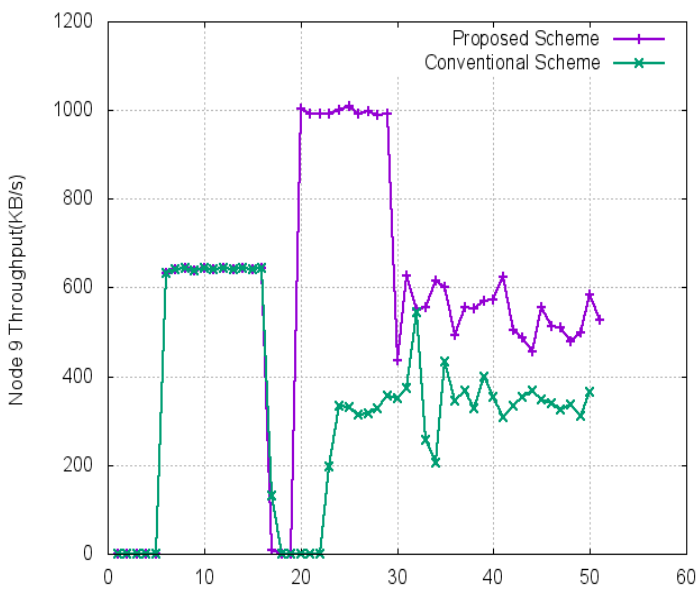

Figure 6. Throughput of UE2 (Node 9) 


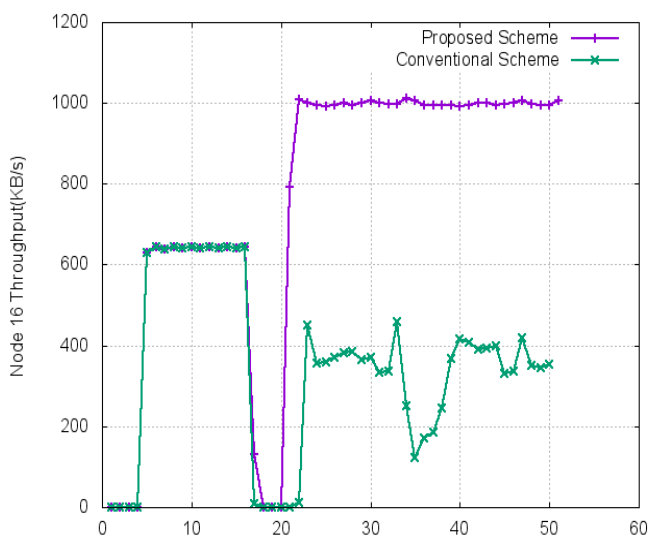

Figure 7. Throughput of UE3 (Node 16)

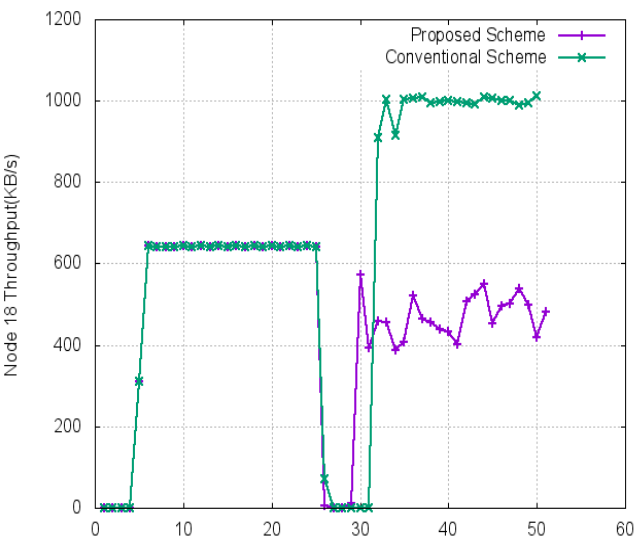

Figure 8. Throughput of UE4 (Node 18)

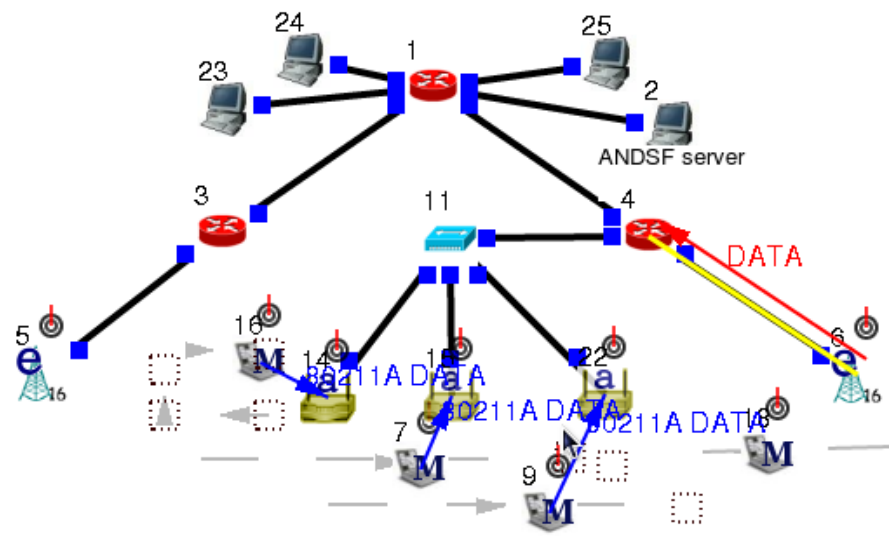

Figure 9. Screenshot of simulator: UE1, 2, 3 are distributed to appropriate APs with proposed scheme (Blue line shows the connection link)

In order to evaluate our proposed scheme, we compare with the conventional WiFi offloading strategy, which is adopted by most of existing smart terminals. As aforementioned, in the conventional WiFi AP selection strategy, if there are several of available APs detected, the AP with the strongest RSS/SINR will be selected and connection will remain until the received sensitive threshold is exceeded. After the connection is broken, the UE detects and associates with alternative point of service. We compare the throughput of UE1, 2, 3, 4 in the proposed case (red line) and the conventional case (green line) shown on Figure [5-8].

At the beginning, all of the UEs are associated with WiMAX BS; therefore, there is no difference in the throughput of UEs. However, Figure [5-8] show that the proposed case has lower service disruption time when UEs and macro-cell BS connections are suddenly disrupted. This can be explained as the proposed scheme can quickly detect connection failure and immediately start the roaming decision procedure. In the conventional case, it takes more time to detect connection failure because the sending data application on the UE has to report to the CM. In addition, the scanning time for available WiFi AP is also longer since the UEs have to scan all WiFi channels to detect available AP. In the proposed scheme, the ANDSF discovery information shortens the scanning process. This result is also mentioned in [11]. As shown in Figure [5-8], the proposed scheme (red line) always takes 1-2s less than the conventional case (green line). In other words, the connection-disrupted time is less compared to the conventional case. 
We also notice the improvement of The UE's overall Quality of Experience (Downlink/Uplink throughput) in the proposed case. The reason for the improvement is that the proposed scheme takes into account the ANDSF's policies, AP's load, RSS to select preferable candidate AP for UE while in the conventional case, only RSS is taken into account for selecting AP. As a result, in the proposed case, the UE selects the AP that has better combined benefit in terms of load and RSS. As shown in Figure 9, the UEs are evenly distributed to available APs so that UEs can achieve better throughput. In the conventional case, the UE1, 2, 3 select same AP1 to connect since it is the nearest one from their location while AP2, 3 remain idle. As shown in Figure [5-7], after transferring to $\mathrm{WiFi}$, the throughput of UE1, 2, 3 using conventional scheme are much lower than the proposed scheme. Moreover, the throughput in the conventional case is more fluctuated due to the contention control mechanism of WiFi AP serving multiple UEs.

At $\mathrm{T}=25 \mathrm{~s}$, when the BS2 connection is disrupted, the proposed scheme also has shorter service disconnected time due to the similar reason. However, the UE4 in the proposed case has lower throughput because it selects the same AP3 in both cases. In the proposed case, from T=30s the AP3 serves both UE2 and UE4 while AP3 only serves UE4 in the conventional case. Although UE4 experiences lower throughput in the proposed case, other UEs can enjoy better throughput and the utilization ratio of $\mathrm{WiFi}$ APs is higher ( $2 / 3$ vs $3 / 3)$.

We can conclude that the proposed scheme outperforms the conventional scheme in terms of reducing disrupted service time, increasing UE's QoE and improving WiFi AP resource utilization.

\section{Conclusion And Future Work}

Our main contribution in this paper is that we proposed a novel monitoring and roaming selection scheme for enhancing cellular and WiFi interoperability. The proposed scheme is aimed at exploiting the advantage of WiFi network to support and backup the cellular network not only in case of congestion but also in unexpected network failure occurrence. In the proposed scheme, we utilized the channel load metric first defined in IEEE802.11k for our proposed scheme. As far as we concerned, this channel load has not been considered in any related work. In addition, we utilize a series of signal level and AP load thresholds to eliminate unqualified candidates, which reduce the processing time and reduce unnecessary delay. We proposed to append signal level threshold into the ANDSF OM WLANSP leaf so that UE can easily fetch this information from ANDSF server. In addition, we also considered the practical implementation of our proposed scheme.

Therefore, we reviewed the state of the art in 3GPP and IEEE and developed our scheme based on the ratified IEEE $802.11 \mathrm{k}$, u and 3GPP TS 23.312 standard so that it can be applicable in future wireless network. In this paper, we also introduced our simulation model of ANDSF and vertical handover. Unlike previous works, our simulation model is an end-to-end model from application to physical layer. We take into account user 's mobility and realistic traffic model. During the simulation process, the ANDSF server and the UE's connection manager entities behave and interact corresponding to real-time network events. By using this simulation model, we evaluated the proposed scheme in a typical heterogeneous network scenario with interworking macro-cell (WiMAX) and WiFi. The result showed that our proposed scheme can quickly detect unexpected network event such as network disruption and effectively assists the UE to find preferable alternative point of service. The connection-disrupted time is reduced and the UE's quality of experience (throughput) is generally improved. In our future work, we will further enhance the proposed scheme and evaluate in more complex network scenario. In this paper, the network is assumed to support only data-like services. Other types of services, such as voice or a combination of different services could also be studied. A more realistic user behavior model could be employed. The robustness of the results can be analyzed by varying some system parameters such as hotspot radius and hotspot position. 
International Journal of Computer Networks \& Communications (IJCNC) Vol.8, No.1, January 2016

\section{REFERENCES}

[1] Doug W, Announcing the Cisco Visual Networking Index Mobile Forecast, 2010-2015, Available online: http://blogs.cisco.com/tag/mobile-data-forecast (Access on December 27th, 2014).

[2] 3GPP. 3rd Generation Partnership Project; Architecture enhancements for non-3GPP accesses (Release 12); Technical Specification 3GPP TS 23.402; March 2014.

[3] 3GPP. 3rd Generation Partnership Project; Access Network Discovery and Selection Function Management Object (Release 12); Technical Specification 3GPP TS 24.312; V12.4.0; March 2014.

[4] IEEE, IEEE Standard for Information Technology- Telecommunications and information exchange between systems- Local and metropolitan area networks-Specific requirements, Part 11: Wireless LAN Medium Access Control (MAC) and Physical Layer (PHY) Standards IEEE Std 802.11 $1^{\mathrm{TM}}$. February, 2012.

[5] Wi-Fi Alliance; Hotspot 2.0 Technical Specification Package (Release 2);v1.0.0.

[6] Kassar, M.; Kervella; B.; Pujolle G. An overview of vertical handover decision strategies in heterogeneous wireless networks, Journal of computer communications, Vol.37, No.10. 2008.

[7] 4G Americas; Integration of Cellular and Wi-Fi Networks; Sep, 2013;

[8] Hugues, S.; Figueiredo, L.; Rabadão, C.; Pereira, A.; Wireless Networks Interoperability-Wifi Wimax Handover. ICSNC'09. Fourth International Conference on, pp. 100-104. IEEE, 2009.

[9] Voyiatzis; Artemios; A survey of delay-and disruption-tolerant networking applications, Journal of Internet engineering 5, no. 1, 2012.

[10] Zekri; Mariem; Badii J.;Djamal Z. A review on mobility management and vertical handover solutions over heterogeneous wireless networks. Computer Communications 35, no. 17, 2055-2068, 2012.

[11] Noroozi, N. ; Abbasfar, A.; Adaptive Load Balancing in LTE-Advanced Networks with Base Station Coordination, NICT, ISSN:1882-5621/13, 2013.

[12] Hagos, D; Kapitza, .R; Study on performance-centric offload strategies for LTE networks, 2013 6th Joint IFIP Wireless and Mobile Networking Conference (WMNC), pp.1-10, April 2013.

[13] Jung, B.; Song, N.O; Sung, D.K. A Network-Assisted User-Centric WiFi-Offloading Model for Maximizing Per-User Throughput in a Heterogeneous Network. Vehicular Technology, IEEE Transactions on 63.4 (2014): 1940-1945.

[14] Lee, J.; Yi, Y; Chong, S.; Jin, Y. Economics of WiFi offloading: Trading delay for cellular capacity. Wireless Communications, IEEE Transactions on 13.3 (2014): 1540-1554.

[15] Bithas, P. S.; Lioumpas, A; Karagiannidis, J.K.; Sharif, S.B. Interference minimization in hybrid WiFi/cellular networks. Computer Aided Modeling and Design of Communication Links and Networks (CAMAD), 2014 IEEE 19th International Workshop on. IEEE, 2014.

[16] IEEE; IEEE Standard for Local and metropolitan area networks. Part 21: Media Independent Handover Services; US; IEEE; 2009.

[17] Meriem, K.; Brigitte, K.; Guy, P.; An overview of vertical handover decision strategies in heterogeneous wireless networks; Computer Communications; v.31 n.10; p.2607-2620; June; 2008.

[18] Omar, K.; Omar, A.; A Survey on Media Independent Handover (MIH) and IP Multimedia Subsystem (IMS) in Heterogeneous Wireless Networks; Springer; vol. 20; no. 2; pp. 215-228; Jun 2013.

[19] IEEE; IEEE Standard for Information Technology- Telecommunications and information exchange between systems- Local and metropolitan area networks- Specific requirements, Part 11: Wireless LAN Medium Access Control (MAC) and Physical Layer (PHY) Standards Amendment 1: Radio Resource Measurement of Wireless LANs, 802.11k-2008.; version 1.2.1; July 2008.

[20] Green Packet Mobile data offload solution using ANDSF. Online available: http://www.greenpacket.com/solutions/dynamic-Wi-Fi-offload.html (Access on December 27th, 2014).

[21] The NCTUns tool. Available online: http://nsl.csie.nctu.edu.tw/nctuns.html. (Access on December 27th, 2014).

[22] S.Y. Wang; P.F. Wang; Y.W. Li; L.C. Lau; Design and Implementation of A More Realistic Radio Propagation Model for Wireless Vehicular Networks over the NCTUns Network Simulator IEEE WCNC 2011; March 28 - 31; 2011.

[23] S.Y. Wang; P.F. Wang; NCTUns 6.0: A simulator for advanced wireless vehicular network research; IEEE Vehicular Technology Conference; May 2010; pp. 1-2. 
International Journal of Computer Networks \& Communications (IJCNC) Vol.8, No.1, January 2016

[24] S.Y. Wang; R.M. Huang; NCTUns Tool for Innovative Network Emulations; a chapter of the Computer-Aided Design and Other Computing Research Developments, ISBN: 978-1-60456-860-8; 2009

\section{AUTHORS}

Nam Nguyen received the B.Sc. degree in Electronics and Telecommunication Engineering from Hanoi University of science and technology in 2007. He received the Master Degree in Information and Communication from Graduate School of Global Information and Telecommunication Studies (GITS), Waseda University, Japan in 2012. Currently, he is a PhD candidate at GITS, Waseda University, Tokyo, Japan. His research interests are in the area of HetNet, Wi-Fi offloading for Cellular N etwork. He is a student member of IEEE since 2011. He received Japan Government Scholarship since 2010.

Takuro Sato received the B.E. and $\mathrm{PhD}$ degrees in electronics engineering from Niigata University, Japan, in 1973 and 1993, respectively. He joined the Research and Development Laboratories, Oki Electric Industry Co., Ltd., Tokyo, Japan in 1973, and he has been engaged in research on PCM transmission equipment, mobile communication, data transmission technology, and digital signal processing technology. He developed Wide-band CDMA system. He has become the Dean of Graduate School of Global Information and Telecommunication Studies, Waseda University since 2012. He has

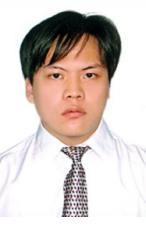
become IEEE fellow since 2012. 